International Mathematical Forum, 2, 2007, no. 24, 1193 - 1206

\title{
On Some Properties of Banach Spaces and Banach Algebras
}

\author{
Yusuf Abilov \\ Odlar Yurdu University \\ Baku, Azerbaijan \\ y.abilov@mail.ru \\ Mehmet Açıkgöz \\ University of Gaziantep, Faculty of Science and Arts \\ Department of Mathematics, 27310 Gaziantep, Turkey \\ acikgoz@gantep.edu.tr
}

\begin{abstract}
It is shown that some questions considered in [1, 2], can be under certain conditions shown to symmetric Banach $\left(C^{*}-\right)$ algebras that allows to solve these questions within the framework of traditional symmetric Banach algebras. It also allows to consider some questions, concerning properties of norms on Banach spaces and Banach algebras.
\end{abstract}

Mathematics Subject Classification: Primary 46A15, Secondary 41A65

Keywords: Separable Banach space, Banach algebra, Symmetric Banach algebras

\section{INTRODUCTION}

During the long period does not weaken interest to questions which concern connection between Banach and Hilbert spaces. One of the basic questions is the known as Banach-Mazur "rotation" problem: If every transitive separable Banach space is a Hilbert space.

Difficultly in one article, even with brief comments to capture all publications and approaches to this problem. However among articles devoted to this problem, it is neccassary to note articles [1,2] in which the review of approaches to this problem, is rather in detail carried out, and also the analysis of this problem is carried out from the point of view of various approaches and decisions of this problem are offered at certain, but weaker conditions. These articles have served as the main reason of to write this article. In article [2], 
this problem is analyzed on the basis of the following approaches to norms in Banach space: Transitivity, Almost-transitivity, convex transitivity, maximality of norm. All these approaches have for an object to weaken strong enough condition of transitivity of norm in Banach space. In this connection in [2] the different approaches based on various methods of the functional analysis are considered and analyzed. To these approaches concern: Isometric one dimensional perturbations of the identity, multiplicative characterization of Hilbert spaces, transitivity of Banach spaces having a Jordan structure. The geometry of convex-transitive Banach spaces.

The purpose of this article-to show that the approach based on the multiplicative characterization of Hilbert spaces, under certain conditions, and more likely formulations, can be considered within the framework of symmetric Banach algebras with unit [3-14]. Thus the same result, but appears some opportunity to look at norm (Theorem 5) a little from other point of view.

\section{The Formulation And the Analysis of A Problem}

In this article, the following notations will be used. $X$-normed space over

$K$ (field of real or complex numbers ); $X^{\prime}$-conjugate (dual ) to $X ; S_{X}$-the unit sphere; $B_{X}$-the closed unit ball; $G:=G(X)$-the group of all surjective linear isometries from $X$ to $X$.

A normed space $X$ is said to be transitive if for every $x, y$ in $S_{X}$ there exists $T$ in $G$ such that $T(x)=y$.

It is well-known that pre-Hilbert spaces are transitive. However exist transitive, but non-separable Banach spaces not being Hilbert spaces. In his book [4] S.Banach raises the following question, called the Banach-Mazur rotation problem.

\section{Problem 1. Is every transitive separable Banach space a Hilbert space?}

This problem remains open to date. The answer is negative if the assumption of separability is removed. The answer is affirmative if the assumption of separability is changed by finite dimensionality (i.e. to strengthen this condition). Now there are many partition decisions of this problem, each of which is based on concrete approaches and methods.

Let's consider in more detail the approach which is based on multiplicative characterization of Hilbert spaces and Banach algebras with unit, possessing very interesting properties.

As it is fairly noted in $[1,2]$, the geometry of norm-unital Banach lgebras at their units is very peculiar, and every real Hilbert space enjoys this peculiarity (at any norm-one element): every real Hilbert space $H$ can be converted into a norm-unital Banach algebra with arbitrarily prefixed unit in the unit sphere of $H$. Therefore it must be expected that Banach spaces possesing "many" points in their unit spheres enjoying such a peculiarity (for instance, almost 
transitive norm-unital Banach algebras ) have to be very "special" and is a Hilbert space. Just these questions will be considered here.

Further is noted, since most properties of the geometry of a norm-unital Banach algebra at its unit are inherited by subspaces containing the unit, in fact consider the following chain of conditions on a norm-one element $e$ of a Banach space $X$ over $K$ :

a) There exists a norm-one bounded bilinear mapping $f: X \mathrm{x} X \rightarrow X$ satisfying $f(e, x)=f(x, e)=x$ for every $x$ in $X$.

b) $m(X, e)=1$, where $m(X, e)$ means the infimum of the set of numbers of the norm $\|f\|$ when $f$ runs over the set of all bounded bilinear mappings $f: X \mathrm{x} X \rightarrow X$ satisfying $f(e, x)=f(x, e)=x$ for every $x$ in $X$.

c) $\operatorname{sm}(X, e)=1$, where $\operatorname{sm}(X, e)$ stands for the infimum of the set of numbers of the form $\max \left\{\|f\|, 1+\left\|L_{e}^{f}-1\right\|, 1+\left\|R_{e}^{f}-1\right\|\right\}$ when $f$ runs over the set of all bounded bilinear mappings from $X \mathrm{x} X$ to $X$.(Here, for $u$ in $X, L_{e}^{f}$ and $R_{e}^{f}$ denote the operators on $X$ given by $x \rightarrow f(u, x)$ and $x \rightarrow$ $f(x, u)$,respectively.)

d) There exists a Banach space $Y$ over $K$ containing $X$ isometrically, together with a norm-one bounded bilinear mapping $f: Y \mathrm{x} X \rightarrow Y$, such that the equality $f(e, y)=f(y, e)=x$ holds for every $y$ in $Y$.

It can be shown that have place $(a) \Longrightarrow(b) \Longrightarrow(c) \Longrightarrow(d)$.

Every norm-one element $e$ in a real Hilbert space $X$ satisfies condition $(a)$ above (with $f(x, y)=(x \mid e) y+(y \mid e) x+(x \mid y) e)$.

An element $e$ of a Banach space $X$ over $K$ acts as a unit on $X$ if $e$ belongs to $S_{X}$ and fulfills condition $(d)$.

It is known [15] that a characterization of real Hilbert spaces in terms of elements which act as units seems to require some kind of "abundance" of such points. In article [1] for real Banach spaces the basic theorem is proved.

Theorem 2.5.([2], Theorem 4.1). A real Banach space $X$ is a Hilbert space if and only if there is a non rare subset of $S_{X}$ consisting of elements which act as units on $X$.

To prove these theorems is used two lemmas.

Lemma 1. The set $U$ of all elements in $X$ which act as units on $X$ is normclosed in $X$.

Lemma 2. Let e act as a unit on $X$, and assume that $E$ is smooth at e. Then $R_{e}$ is an $L_{2}-$ summand of $E$.

For a case complex Banach spaces the approach, on a basis the numerical range, $V(X, e, x)$, of $x$ relative to $(X, e)$ is considered. The basic approach, certainly, is the following. For a complex Hilbert space of dimension $\geq 2$ has no element acting as a unit. Because of it consider following definition.

An element $e$ of a Banach space $X$ over $K$ acts weakly as a unit on $X$ whenever $e$ belongs to $S_{X}$ and fulfills condition $(*)$ which follows: 
(*) There exists a Banach space $Y$ over $K$ containing $X$ isometrically, together with a norm-one bounded bilinear mapping $f: X \times X \rightarrow Y$,

such that the equality $f(e, x)=f(x, e)=x$ holds for every $x$ in $X$.

As may be seen the condition $(*)$ is similar to a condition $(d)$ which was considered above, but with that difference, that $X$ is changed on $Y$, that is very essential. And after that Lemma 4.3 and Theorem 4.4 is given which are main results of this article.

Lemma 3. For a norm-one element e of a Banach space $X$ over $K$, the following assertions are equivalent:

1) e acts weakly as a unit on $X$.

2) The equality $\|e \otimes x+x \otimes e\|_{\pi}=2\|x\|$ holds for every $x$ in $X$, where $\|\cdot\|_{\pi}$ means projective tensor norm.

3) There exists a Banach space $Y$ over $K$, together with a norm-one bounded symmetric bilinear mapping $f: X x X \rightarrow Y$, such that the equality $\|f(e, x)\|=$ $\|x\|$ holds for every $x$ in $X$.

Theorem 4. For a Banach space $X$ over $K$, the following assertions are equivalent:

1) $X$ is a Hilbert space.

2) Every element of $S_{X}$ acts weakly as a unit on $X$.

3) There is a dense subset of $S_{X}$ consisting of elements which act weakly as unit on $X$.

4) The equality $\left\|x_{1} \otimes x_{2}+x_{2} \otimes x_{1}\right\|_{\pi}=2\left\|x_{1}\right\|$. $\left\|x_{2}\right\|$ holds for all $x_{1}, x_{2}$ in $X$, where $\|\cdot\|_{\pi}$ means projective tensor norm.

5) There exists a Banach space $Y$ over $K$, together with a symmetric bilinear mapping $f: X x X \rightarrow Y$, such that the equality $\left\|f\left(x_{1}, x_{2}\right)\right\|=\left\|x_{1}\right\| \cdot\left\|x_{2}\right\|$ holds for all $x_{1}, x_{2}$ in $X$.

Proofs are based on symmetry property of bilinear mapping $f: X \mathrm{x} X \rightarrow Y$ in the complex Banach space.

Proof. (5) $\Longrightarrow(1)$ (basic result). Assume that (5) holds. Then, for $x, z$ in $S_{X}$, we have

$4=4\|f(x, z)\|=\|f(x+z, x+z)-f(x-z, x-z)\| \leq\|x+z\|^{2}+\|x-z\|^{2}$.

Therefore, by Schoenberg's theorem, $X$ is a Hilbert space.

First, it is necassary to note that conditions $(*),(5)$ of Theorems 4.4, (3) of Lemma 4.3 are necassary and sufficient to set in Banach space $X$ continuous operations of multiplication and involution (M. Burgos, Non-Associative unitary Banach algebras, 2005).

Second, from this proof are not clear properties and connection of norms Banach and Hilbert spaces. It is necessary to note, that these conditions strong enough for norm.

Third, it is not visible the dependence of norm from functionals of the conjugate space $X$. It is may be shown, that from condition (*), Lemma 4.3 and 
Theorem 4.4 follows, that on complex Banach space $X$ is possible to set multiplication and involution operations that converts $X$ to symmetric Banach algebra.

\section{The Basic notions and Results of Symmetric Banach}

\section{Algebras}

All further concepts and results can be found in M.Najmark's works [ 3-15 ].

$R$ is called normed algebra if:

1) $R$ is algebra,

2) $R$ is normed space,

3) For $\forall x, y \in X,\|x, y\|=\|x\| \cdot\|y\|$,

4) If in $R$ exist unit element $e$ then $\|e\|=1$.

If $R$ is complete normed space (i.e. Banach space) then $R$ is called Banach algebra.

It is necassary to note, that in M. Najmark's works $[1-5,7,11]$ a Banach algebra refers to as a Banach ring.

$R$ is called symmetric algebra, if

1) $R$ is an algebra,

2) In $R$ operation, which to each element $x$ from $R$ is determined

puts in conformity an element $x^{*}$ from $R$ so, that are executed the following conditions:

a) $(\lambda x+\mu y)^{*}=\bar{\mu} x^{*}+\bar{\mu} y^{*}$

b) $x^{* *}=x$,

c) $(x y)^{*}=y^{*} x^{*}$.

$x \rightarrow x^{*}$ we shall name this operation involution, and elements $x^{*}$ and $x$ are conjugate to each other. The element $x$ is called hermitian, if $x^{*}=x$. Any element of a kind $x^{*} x$ is hermitian. Unit $e$ is hermitian element. Everywhere further if it is not stipulated specially, are considered symmetric Banach $\left(C^{*}-\right)$ algebras.

The mapping $\varphi: R \rightarrow P$ is called symmetric homomorphism if:

1) $\varphi$ is homomorphism,

2) from $x \rightarrow y$ follows, that $x^{*} \rightarrow y^{*}$.

If $\varphi: R \rightarrow P$ is isomorphism, then $\varphi$ is symmetric isomorphism. Ideal $I$ (left, right and two-sided) is called symmetric if from $x \in I$ follows that $x^{*} \in I$. Symmetric ideal is automatically two-sided. Quotient algebra $R / I$ is too symmetric algebra.

Linear functional $f$ is called real if functional $f$ takes real values on all hermitian elements of algebra $R$. If $f$ is real functional then $f\left(x^{*}\right)=\overline{f(x)}$ for 
all $x \in R$. Linear functional $f$ is called positive if $f\left(x^{*} x\right)>0$ for any $x \in R$. For arbitrary positive functional $f$ in symmetric algebra $R$

$$
\begin{gathered}
f\left(y^{*} x\right)=\overline{f\left(x^{*} y\right)} \\
\left\|f\left(y^{*} x\right)\right\|^{2}=\left\|f\left(y^{*} y\right)\right\| \cdot\left\|f\left(x^{*} x\right)\right\|
\end{gathered}
$$

Proposition 5. Any positive functional $f$ in symmetric algebra $R$ with unit $e$ is real and

$$
\|f(x)\|^{2}=f(e) \cdot f\left(x^{*} x\right)
$$

Proof. If suppose that $y=e$ the proof follows from formula (2). $R$ is called symmetric Banach algebra if

1) $R$ is Banach algebra,

2) $R$ is symmetric algebra,

3) $\left\|x^{*}\right\|=\|x\|$.

From condition (3) follows that involution operation is continuous.

Example 1. $B(X)$ is the set of all bounded linear operator in Banach space $X . B(X)$ is complete normed space and the norm is operator norm. In the $B(X)$ is defined multiplication as multiplication of operators, i.e. $\|A B\| \leq$ $\|A\| .\|B\|$. Consequently, $B(X)$ is Banach algebra. If $X$ is Hilbert space then $B(X)$ is symmetric algebra if under involution understand transition to the conjugate operator.

Proposition 6. Any positive functional $f$ in symmetric Banach algebra $R$ with unit $e$ is bounded functional and satisfy condition

$$
\|f(x)\|=\|f(e)\| \cdot\|x\|
$$

Proof. can be found in [11].

Proposition 7. The norm $\|f\|$ of positive functional $f$ in symmetric Banach algebra $R$ with unit e is equal to $f(e)$, i.e.

$$
\|f\|=f(e) .
$$

Proof. From inequality (1) follows that $\sup _{\|x\|=1}\|f(x)\| \leq f(e)$. On the other side this inequality is reached for $x=e$.

The representation of algebra $R$ is called any homomorphism of algebra $R$ into algebra $B(H)$ of linear bounded operators in vector space.

The symmetric representation of symmetric algebra $R$ is called symmetric homomorphism $x \rightarrow A_{x}$ of algebra $R$ into algebra $B(H)$.

The representation is calle continuous if homomorphism of representation is continuous.

The representation is called cyclic if in space $H$ there is the vector $\xi_{0}$ such that the set of all vectors $A_{x} \xi_{0}$ is dence in $X$. The vector $\xi_{0}$ and space $H$ is called 
cyclic for representation $x \rightarrow A_{x}$. Two representation $x \rightarrow A_{x}$ and $x \rightarrow B_{x}$ in space $C$ is called equivalent if there exists isometric mapping $U: H \rightarrow H$ under which operator $A_{x}$ transform to operator $B_{x}$ for all $x$ in $H$. On the other words if $U: H \rightarrow H$ is isometric mapping and if $\xi^{\prime}=U \xi$ then $B_{x} \xi^{\prime}=U A_{x} \xi$. Consequently, $B_{x} U \xi=U A_{x} \xi$ for all elements $\xi$ in $H$, i.e. $B_{x} U=U A_{x}$. The subspace $H_{1} \subset H$ is called invariant if any $x \in H_{1}$ transform by all operators $A_{x}$ again in vectors of $H_{1}$. If $H_{1} \subset H$ is closed invariant subspace then all operators $A_{x}$ can be considered as operators in $H_{1}$. If $H_{1} \subset H$ is invariant then its orthogonal complement is invariant. It is also the convex hull of invariant subspaces is a invariant subspace.

Let us given the set of representations $x \rightarrow A_{x}^{(\alpha)}, \alpha \in U$, of symmetric algebra $R$ into $H_{\alpha}$. Let us

$$
\left\|A_{x}^{(\alpha)}\right\| \leq C_{x}
$$

where $C_{x}$-is independent of $\alpha$. Denote by $H$ the direct sum of spaces $H_{\alpha}$, i.e. the set of all elements $\xi \in\left\{\xi_{\alpha}\right\}$ such that

$$
\sum_{\alpha}\left\|\xi_{a}\right\|^{2} \leq+\infty
$$

Let us assume that $A_{x} \xi=\left\{A_{x}^{(\alpha)} \xi_{a}\right\}$. By (6), $A_{x}$ is bounded operator in $H$ and mapping $x \rightarrow A_{x}$ is representation in space $H$. This representation $x \rightarrow A_{x}$ is called direct sum of original representations $x \rightarrow A_{x}^{(\alpha)}$. Any representation is direct sum of cyclic representation.

\section{BAsic Results}

Show how can be described symmetric representation on base of positive functionals.

Let us $x \rightarrow A_{x}$ is representation of symmetric algebra $R$ in space $H$ and let us $\xi_{0}$ is non zero in space $H$. Assume that

$$
f(x)=\left(A_{x} \xi_{0}, \xi_{0}\right) .
$$

Show that $f$ is positive functional.Really,

$$
f\left(x^{*} x\right)=\left(A_{x^{*} x} \xi_{0}, \xi_{0}\right)=\left(A_{x}^{*} \xi_{0}, \xi_{0}\right)=\left(A_{x} \xi_{0}, A_{x} \xi_{0}\right) \geq 0 .
$$

Theorem 8. Any symmetric representation of Banach algebra $R$ is continuous and

$$
\left\|A_{x}\right\| \leq\|x\| .
$$

Proof. can be found in [M.N.].

Further it is supposed that all considered representations are cyclic.

Let us $x \rightarrow A_{x}$ and $x \rightarrow B_{x}$ is cyclic representations in space $R$. Assume that

$$
f(x)=\left(A_{x} \xi_{0}, \xi_{0}\right), \quad f^{\prime}(x)=\left(A_{x} \xi_{0}^{\prime}, \xi_{0}^{\prime}\right)
$$


where $\xi_{0}$ and $\xi_{0}^{\prime}$ are cyclic vectors of these representations.

Proposition 9. If $f(x)=f^{\prime}(x)$ for all $x$ in $R$ then representations $x \rightarrow A_{x}$ and $x \rightarrow B_{x}$ is equivalent.

The proof is simple and therefore is omitted.

This proposition show that cyclic representation $x \rightarrow A_{x}$ with cyclic vector $\xi_{0}$ uniquely with accuracy up to equivalency is defined

by positive functional $f(x)=\left(A_{x} \xi_{0}, \xi_{0}\right)$. Then arises the question, is it true that for any positive functional $f$ exists

representation $x \rightarrow A_{x}$ such that $f(x)=\left(A_{x} \xi_{0}, \xi_{0}\right)$ ? The answer is affirmative in the case Banach symmetric algebra $R$ with unit $e$.

Let us $f$ is positive functional. Involve in $R$ the hermitian form $(x, y)=f\left(y^{*}, x\right)$. At the same time can occur, that for some elements $x$ in $R$ will be $(x, x)=f\left(x^{*}, x\right)=0$. Each such element call equivalent zero and write up $x^{-} 0$.

Denote by $I_{l}^{f}$, the set of all elements $x$ in $R$ which is equivalent zero.

Proposition 10. $I_{l}^{f}$ is left ideal.

The proof is simple and therefore is omitted.

Denote by $H^{\prime}$ quotient space $R / I_{l}^{f}$, by $\xi, \eta, \ldots$ the elements of this space, i.e. quotient classes by ideal $I_{l}^{f}$. If $\xi, \eta$ is two such classes and $x, y$ are their representatives in these classes then assume

$$
(\xi, \eta)=(x, y)=f\left(y^{*} x\right) .
$$

Expression $(\xi, \eta)$ is independent from selection representatives $x, y$. Really, if, for example, $x^{\prime}, x^{\prime \prime}$ are two elements of class $\xi$, then $x^{\prime}-x^{\prime \prime}-0$, consequently, $\left(x^{\prime}, y\right)-\left(x^{\prime \prime}, y\right)=\left(x^{\prime}-x^{\prime \prime}, y\right)=f\left(y^{*}\left(x^{\prime}-x^{\prime \prime}\right)\right)=0$.

It is easy to verify that $(\xi, \eta)$ possesses all of properties of scalar product, i.e.

i) $(\xi, \eta)=\overline{(\eta, \xi)}$,

ii) $(\xi \eta+\mu \eta, \xi)=\lambda(\xi, \xi)+\mu(\eta, \xi)$,

iii) $(\xi, \xi)>0$ if $\xi \neq 0$.

The property (ii) is evident. Property (i) follows from reality of functional $f$. consider more detailly property (iii).

Let $x$ is element of class $\xi$. Since $f$ is positive functional then $(\xi, \eta)=$ $f\left(x^{*} x\right)>0$. If, in the same time, $(\xi, \eta)=f\left(x^{*} x\right)=0$ then $x^{-} 0$, i.e. $\xi=0$. Thus, in $H^{\prime}$ is built scalar product. Completion $H^{\prime}$ with respect to this scalar product denote by H.H is the Hilbert space. Denote by $\xi=[x]$ the class with representative $x$, and by $\eta=\left\{x_{0} x\right\}$ the class with representative $x_{0} x$. Since $I_{l}^{f}$ is left ideal then $\eta$ doesn't depend from selection representative $x$ of class $\xi$. Assume that

$$
A_{x_{0}} \xi=\left[x_{0} x\right]
$$


then will get linear operator in space $H^{\prime}$. Let us show, that it is bounded. By definition of scalar product in $H^{\prime}$

$$
\left\|A_{x_{0}} \xi\right\|^{2}=\left(A_{x_{0}} \xi, A_{x_{0}} \xi\right)=\left(x_{0} x, x_{0} x\right)=f\left(x^{*} x_{0}^{*} x_{0} x\right) .
$$

Assume $f_{1}(y)=f\left(x^{*} y x\right)$. $f_{1}(y)$ is positive functional. In fact, $f_{1}\left(y^{*} y\right)=$ $f\left(x^{*} y^{*} y x\right)=f\left((x y)^{*} y x\right) \geq 0$. Applying to $f_{1}(y)$ inequality (4) proposition 2 getting $\left\|f_{1}(y)\right\| \leq f_{1}(e)$. $\|y\|$. If assume that $y=x_{0}^{*} x$ then $\left\|f_{1}\left(x_{0}^{*} x x_{0}\right)\right\| \leq$ $f_{1}(e)\left\|x_{0}^{*} x_{0}\right\| \leq f_{1}(e)$. $\left\|x_{0}\right\|^{2}$, i.e. $\left\|f\left(x^{*} x_{0}^{*} x_{0} x\right)\right\| \leq f\left(x^{*} x_{0}\right)\left\|x_{0}\right\|^{2}$. As a result of the fact that (11) it is mean that

$$
\left\|A x_{0} \xi\right\|^{2} \leq\left\|x_{0}\right\|^{2} \cdot\|\xi\|^{2} \text {. }
$$

Thus, $A x_{0}$ is bounded operator in $H^{\prime}$ and its norm doesn't exceed $\left\|x_{0}\right\|$. Consequently,this operator can be extended and unique way to bounded operator in space $H$. Thus the norm $\left\|A x_{0}\right\|$ doesn't change. Consequently, inequality (12) remains in force and for the norm of operator $A x_{0}$ in space $H$. The mapping $x \rightarrow A_{x}$ is the representation. It can be proved by not difficult transformation and now show that this representation is cyclic. Let us $\xi_{0}$ is the class including element $x_{0}$. Consequently, the set of all vectors of type $A_{x} \xi_{0}, x \in R$, coincides with the set $H^{\prime}$ of all classes. Since $H^{\prime}$ is dense in $H$ then from here follows that $\xi_{0}$ is cyclic vector. Now find at last, what is the value of $\left(A_{x} \xi_{0}, \xi_{0}\right)$. The class $\xi_{0}$ including unit element e, thus

$$
\left(A_{x} \xi_{0}, \xi_{0}\right)=(x e, e)=f\left(e^{*} x e\right)=f(x) .
$$

Consequently, is proved the following theorem.

Theorem 11. To each cyclic representation $x \rightarrow A_{x}$ of symmetric Banach algebra $R$ with cyclic vector $\xi_{0}$ corresponds positive functional $f(x)=\left(A_{x} \xi_{0}, \xi_{0}\right)$. By functional $f(x)$ representation $x \rightarrow A_{x}$ is defined uniquely with accuracy up to equivalancy. Conversely, to each positive functional $f(x)$ in symmetric $B a-$ nach algebra $R$ with unit element e corresponds cyclic representation $x \rightarrow A_{x}$ such that $f(x)=\left(A_{x} \xi_{0}, \xi_{0}\right)$.

It is clear that both spaces $H$ and $H^{\prime}$ are symmetric Banach algebras, and consequently, Banach spaces with added structure and involution operation. If now we consider $R$ and $H$ as Banach spaces and take into acount that $H^{\prime}=R$ $/ I_{l}^{f}$ then becomes clear that mapping $\tau: H^{\prime} \rightarrow R(\tau: H \rightarrow R)$ isometrically include $H$ in $R$ as in condition (*) of elements which act weakly as unit [1,2].

The symmetric bilinear mapping $f: X x X \rightarrow Y$, we define in form (9)-(10). The property (3) of lemma 4.3.(i.e. $\|f(e, x)\|=\|x\|$ ) and the property $(*)$ (i.e. $f(e, x)=f(x, e)=x)$ follow from equality (13) as class $\xi_{0}$ including unit element e contains, in the same time, all element equivalent to $e$ and thus only these elements constitute a set which is dense in $S_{x}$. It becomes evidently if in (13) instead of unit $e$ in class $\xi_{0}$ select any other element $x$ equivalent to e, i.e. $\left(A_{x} \xi_{0}, \xi_{0}\right)=\left(x x_{0}, x_{0}\right)=f\left(x_{0}^{*} x, x_{0}\right)=f(x)$. Thus the set of elements in $X$ which act as unit $e$, in fact, are elements consituting class $\xi_{0}$ of unit element e in $X$. 
Taking into account their importance, let us consider irreducible representations. Representation $x \rightarrow A_{x}$ is called irreducible if in space $H$ does not exist closed invariant subspace, distinct from (0) and all $H$.

It is known, that any representation in one-dimensional space is irreducible. Therefore will be considered representations for dimension $n>1$.

Proposition 12. Representation $x \rightarrow A_{x}$ in space $H$ is irreducible, if and only if, any vector of space distinct from zero is cyclic vector of this representation.

Proof. Let us $x \rightarrow A_{x}$ is irreducible. For $\xi \in H, \xi \neq 0$, closed subspace streched (convex hull) on vectors $A_{x} \xi, x \in R$ is invariant subspace coincides with (0) or all $H$. But first case is impossible since then one-dimensional space $\{\alpha, \xi\}$ is invariant and that is why coincide with $H$, i.e. $A_{x}=0$ in $H$. Conversely, if representation $x \rightarrow A_{x}$ is reducible and ....-distinct from (0) and all $H$ closed invariant subspace in $H$, then there isn't vector $\xi \in H$ which is cyclic for representation $x \rightarrow A_{x}$ in $H$.

Proposition 13. Representation $x \rightarrow A_{x}$ in space $H$ is irreducible, if and only if, any any bounded linear operator permutable with all representation operators $A_{x}$ is multiple of one, i.e. multiple of unit operator.

Proof. can be found in $[11,14]$

Now will be presented including symmetric algebra into algebra of operators. Let us $R$ is symmetric algebra. Denote by $F_{R}$ the set of all positive functional in $R$. Evidently, $F_{R}$ is not change if in $F_{R}$ in some way to introduce (to give) the norm $\|x\|$ so that $R$ became normed symmetric space. Really, the definition of positive functional doesn't depend on the norm in $R$. However at transition from algebra $R$ to its completion $\widetilde{R}$ by this norm the set $F_{R}$, as a matter of fact, can change. In other words, not any positive functional in $R$ can be extended to positive functional in $\widetilde{R}$. In [14] is given such example of algebra $R$ of all polynomials $p(t)$ with complex coefficients.

The norm $\|x\|$ in symmetric algebra $R$ with unit $e$ is called regular if any positive functional $f$ in $R$ can be extended to positive functional $\widetilde{f}$ in $\widetilde{R}$. In this case $\widetilde{f}$ is positive functional in complete algebra $R$ with unit e. Then as a matter of fact (4) proposition 2

$$
\|\widetilde{f}(\widetilde{x})\| \leq \tilde{f}(e)\|\widetilde{x}\|=f(e)\|\widetilde{x}\| .
$$

Particularly,

$$
\|f(x)\| \leq f(e)\|x\|
$$

Consequently, the functional $f(x)$ is continuous in $R$ and because can be extended up to $\widetilde{f}$ in its completion $\widetilde{R}$ by a unique way. Then take place the following:

To extend positive functional $f$ in $R$ up to positive functional $\widetilde{f}$ in $\widetilde{R}$, it is necessary and sufficient that the condition (14) was fulfilled. The element $x$ of 
symmetric algebra $R$ with unit $e$ is called bounded if for all positive functional $f$ in $R$ take place $f\left(x^{*} x\right) \leq C f(e)$, where $C$-the constant.

In symmetric Banach algebra $R$ with unit e, all elements are bounded. Really, according to inequality $(14), f\left(x^{*} x\right) \leq f(e)\left\|x^{*} x\right\|$ for all positive functional $f$ in $R$. If symmetric algebra $R$ with unit e exists regular norm the all elements of $R$ bounded. This proposition is consequence from inequality (14) too. Let us $R$ is symmetric algebra with unit e. Suppose that there is at least one positive functional $f$ in $R$ doesn't equal equivalently to zero. An element $x \in R$ is called generalized zero and write $x \approx 0$ if $f\left(x^{*} x\right)=0$ for all positive functional $f$ in $R$.

Present several facts without of proof:

1) The element $x \in R$ is generalized if and only if $f(y x)=0$ for all positive functional $f$ and all elements $y \in R$.

2) The unit e is not generalized zero.

3) The element $x_{0} \in R$ is generalized zero if and only if $f\left(x_{0}\right)=0$ for all positive functional $f$.

4) If $x \approx 0$ then also $x^{*} \approx 0$.

The set $I$ of all generalized zeros in $R$ is symmetric and therefore two-sided ideal. This ideal $I$ is called reducing ideal, and the quotient algebra $R^{\prime}=R /$ $I$ reducible algebra.

If, in particular, $I=(0)$, then algebra $R$ is reducible. Algebras $R^{\prime}$ and $R$ possess one and the same set of positive functionals $f$.In reducible algebra $R^{\prime}$ for any element $x^{\prime} \neq 0$ there is positive functional $f$ such that $f\left(x^{\prime *} x^{\prime}\right) \neq 0$.

And now define minimal regular norm [ 8, 9, 11].

Theorem 14. If in reducible algebra $R$ there is regular norm then in reducible algebra $R$ there is minimal regular norm. The completion algebra $R$ by this minimal regular norm is entirely isomorphic some algebra of operators in Hilbert space.

Proof. Let $\|x\|_{1}$ is some regular norm in $R$, and $R_{1}$-completion of algebra $R$ by this norm. Any positive functional $f$ in $R$ can be extended to positive functional in $R_{1}$. This functional also denotes by $f$. To this functional there corresponds representation of algebra $R_{1}$. Denote this representation by $(x) \rightarrow$ $A_{x}^{(f)}$. Further let us $x \rightarrow A_{x}$ is direct sum of all representations by $f$. According to inequality (8) Theorem 1

$$
\left\|A_{x}\right\| \leq\|x\|_{1}
$$

where $\left\|A_{x}\right\|$ is norm of operator $A_{x}$.

It is significiant that $\left\|A_{x}\right\|$ for all $x \in R$ is entirely defined by the set (resource) of positive functionals in $R$ and therefore doesn't from selection of regular norm $\|x\|_{1}$.

The mapping $x \rightarrow A_{x}$ is isomorphism of algebra $R$. Really, suppose $A_{x}=0$. Then also $A_{x^{*} x}=A_{x^{*}} A_{x}=0$. From here cosequent that also $A_{x^{*} x}^{(f)}=0$, i.e. $\left(A_{x^{*} x}^{(f)} \xi, \eta\right)=0$ for any vectors $\xi, \eta$ from corresponding space $H^{f}$. Suppose, in 
particular, that $\xi=\eta=\xi_{0}$, where $\xi_{0}$ is the class including unit of $R$. Then is obtained that $f\left(x^{*} x\right)=0$ for positive functional in $R$. Since $R$ is reducible algebra then $x=0$.

So $x \rightarrow A_{x}$ is isomorphism of algebra $R$. Introduce in the algebra $R$ norm by formula $\|x\|=\left\|A_{x}\right\|$. Evidently algebra $R$ with norm $\left\|A_{x}\right\|=\|x\|$, and consequently and its completion $\widetilde{R}$ by this norm entirely isomorphic to some algebra of operators in Hilbert space (namely, algebra of all operators $\left.A_{x}\right)$.

And now show that $\|x\|$ is regular norm. Let $f(x)$ is positive functional in $R$. Then for any element $x \in R$

$$
\|f(x)\|=\left\|A_{x}^{(f)} \xi_{0}, \xi_{0}\right\| \leq\left\|A_{x}^{(f)}\right\| \cdot\left\|\xi_{0}\right\|^{2}=\|x\| f(e) .
$$

This inequality shows that functional $f(x)$ is continuous in sense of norm $\|x\|$. Consequently this functional can be extended and thus by uniquely way up to positive functional in completion $\widetilde{R}$ of algebra $R$ by norm $\|x\|$. It is means that $\|x\|$ is regular norm.

It can be shown that for an existing a regular norm in the reducible algebra $R$ it is necassary and sufficient that all elements of algebra $R$ were bounded.

The positive functional $f$ is called indivisible if any functional $f_{1}$ subordinate to functional $f$ is multiple to it, i.e. $f=\lambda f_{1}$.

The cyclic representation $x \rightarrow A_{x}$ irreducible if and only if determining this representation positive functional $f(x)=\left(A_{x} \xi_{0}, \xi_{0}\right)$ is invidisible.

Let $R$ is symmetric Banach algebra with unit e.Denote by $H$, the set of all hermitian elements of algebra $R$. Evidently, $H$ is real Banach algebra. According to inequality (4) proposition 2 any positive functional $f$ can be considered as bounded linear functional in $H$ with $\|f\|=f(e)$. Therefore $f$ can be considered as element of conjugate space $H^{*}$. Denote by $K$, the set positive functionals of unit ball $Q$ in $H^{*}$. It can be proved that $Q$ is compact in weak topology on $H^{*}$, because $Q$ is the set of all linear functional $f \in H^{*}$ and satisfying inequality $\|f(x)\| \leq p(x)$, where $p(x)=\|x\|$. Since the function $F(f)=f(e)$ is continuous, and the set of all positive functional $f$ is closed in $H^{*}$ then $K$ is closed subset of compact set $Q$, and consequently $K$ is compact.

At last $K$ is convex. Indeed, if $f_{1}, f_{2} \in K$, i.e. $f_{1}(e)=f_{2}(e)=1$ then $f=t . f_{1}+(1-t) f_{2}$ for $0 \leq t \leq 1$ is positive functional and $f(e)=1$, consequently, $f \in K$.

Proposition 15. The positive functional $f$ satisfying condition $f(e)=1$ is indivisible if and only if this functional is extremal (extreme) point of set $K$.

The proof is not difficult and can be found in [5, 6, 14].

Now, consider some of the set of irreducible representation of symmetric Banach algebra $R$. Two equivalent irreducible representations will not be considered as different. This set of irreducible representations is called complete if for any element $x_{0} \neq 0$ of algebra $R$ in this set there is irreducible representation $x \rightarrow A_{x}$ such that $A_{x} \neq 0$. 
Theorem 16. The set of all irreducible representations $x \rightarrow A_{x}$ of symmetric Banach reducible algebra $R$ constitute complete set.

Proof. Let $R$ is a Banach algebra, and $x_{0} \neq 0$ is the element of this algebra. By the definition of reducible algebra there is the positive functional $f_{1}(x)$ such that $f(e)=1$ and $f_{1}\left(x_{0}^{*} x_{0}\right)>0$.

Let us further $P_{x_{0}^{*} x_{0}}$ is supporting hyperplane of $K$ defining by element $x_{0}^{*} x_{0}$. It is not difficult to prove that $P_{x_{0}^{*} x_{0}}$ contains extremal point $f_{0}$. Take place

$$
f_{0}\left(x_{0}^{*} x_{0}\right)=\max _{f \in K} f\left(x_{0}^{*} x_{0}\right) \geq f_{1}\left(x_{0}^{*} x_{0}\right)>0 .
$$

On the other hand, according to proposition 1 , any extremal point $f_{0}$ of the set $K$ is indivisible positive functional. This functional define irreducible representations $x \rightarrow A_{x}$ of algebra $R$ and at that $f_{0}(x)=\left(A_{x} \xi_{0}, \xi_{0}\right)$, where $\xi_{0}$-some vector in space of representation. In particular, on account of (2)

$$
\left\|A_{x} \xi_{0}\right\|^{2}=\left(A_{x_{0}^{*} x_{0}} \xi_{0}, \xi_{0}\right)=f_{0}\left(x_{0}^{*} x_{0}\right)>0 .
$$

Consequently, $A_{x_{0}} \neq 0$ and theorem is proved.

Now we shall pass to the basic theorem.

Let us given Banach space $R$, which is equipped by structure of symmetric algebra. In fact, it can be considered Banach space without condition of symmetry. It follows from the Corollary 4, 4, $\mathrm{V}[16]$ where is proved that any Banach space $X$ isometrically isomorphic to some closed suspace of Banach space $C(\Lambda)$ of continuous bounded functions on some bicompact Hausdorff space $\Lambda$. Suppose that $\Lambda$ is unit sphere $S_{X^{*}}$ in conjugate space $X^{*}$. Now if we take into account that $C\left(S_{X^{*}}\right)$ is Banach algebra and isometric isomorphism $\tau: X \rightarrow X^{\prime}$, where $X^{\prime}$ is closed subspace (linear variety) of space $C\left(S_{X^{*}}\right)$ then it is clear that it can be given the structure of Banach algebra on the Banach space $X$.

Theorem 17. Any Banach space $R$ which is equipped with structure of symmetric Banach algebra is Hilbert space.

Proof. Let Banach space $R$ is equipped with structure of symmetric Banach algebra. Consider the set $K$ of all positive functional $f$ from conjugate space $R^{*}$ satisfying condition $f(e)=1$ and $\|f\|=1$. As was shown above, the set $K$ is closed subset of unit ball $Q$ and therefore is from proposition 11 follows that positive functional $f$ satisfying condition $f(e)=1$ is indivisible if functional $f$ is extreme point of the set and from Theorem 6 follows that if functional is indivisible then one define cyclic irreducible representation. Here two cases : 1) $R$ is irreducible symmetric Banach algebra; 2) $R$ is symmetric reducible Banach algebra.

If $R$ is symmetric irreducible Banach algebra then as was noted above the set of all generalized zeros constitutes symmetric ideal $I$. Then instead Banach algebra $R$ will be considered the quotient algebra $R^{\prime}=R / I$ which is symmetric Banach algebra too. 
Let $R$ is reducible symmetric Banach algebra. Consider arbitrary point $x$ in $R$. It is known (from above) that in reducible Banach algebra $R$ for any $x_{0} \neq 0$ exists positive functional $f$ such that $f\left(x_{0}^{*} x_{0}\right) \neq 0$. Then in the point $x_{0}^{*} x_{0}$ (Theorem 7) it can be built functional $f_{0}$ such that $\left(A_{x} \xi_{0}, \xi\right)=f_{0}(x)$ and $A_{x} \neq 0$. Then on base of Theorem 2 it can be built Hilbert space $H^{f}$ in which is determined the cyclic operator of representation $x \rightarrow A_{x}$. Similarly for all points $x \in R$ it can be built Hilbert spaces and operator of representation $x \rightarrow A_{x}$ by corresponding positive functional $f$. Then the direct sum $H$ of Hilbert spaces $H^{f}$ is Hilbert space. From theorem 3 follows that the norm $\|x\|=\left\|A_{x}\right\|$ in $H$ does not depend from norm $\|x\|_{1}$ of Banach space $R$. This

norm $\|x\|=\left\|A_{x}\right\|$ is entirely defined by norm of the set functional in the conjugate space $R^{*}$ and therefore is minimal regular norm.

\section{REFERENCES}

[1] J. Becerra and A. Rodriguez, Isometric reflections on Banach spaces after a paper of A. Skorik and M. Zaidenberrg, Rocky Mountain J. Math. 30 (2000) 63-83.

[2] J. Becerra and A. Rodriguez, Transitivity of the norm on Banach spaces, Extracta Math.17 (2002) 1-58.

[3] Gelfand, I.M., Naimark, M. A., On the imbedding of normed rings into the ring of operators in Hilbert space, mat.sb.12(1943), 197-213.

[4] Gelfand, I.M., Naimark, M. A., Unitary representations of the group of linear transformations of the straight line. C.R. (Doklady) Acad.sci. URSS (N.S) 55, (1947), 571-574.

[5] Gelfand, I.M., Naimark, M. A., Normed rings with involutions and their representations,(Russian)Izvestiya Akad. Nauk SSSR. Ser. Mat.12, (1948), 445-480.

[6] Naimark, M. A., Positive defined operator functions on commutative group, proceedings of the academy of sciences of USSR, Ser. Mat. 7(1943), 237-244.

[7] Naimark, M. A., Ring with involution, UMN USSR 3(1948), 62-145.

[8] Naimark, M. A., Ring of operators in Hilbert space, UMN USSR 4(1949), 83-147.

[9] Naimark, M. A., About one problem of the theory of rings with involution, UMN USSR 6(1951), 162-164.

[10] Naimark, M. A., About the description of all unitary representations of complex classical groups, I. Mat., sb. 35(1954), 317-356.

[11] Naimark, M. A., The normalized rings, Gostexizdat, M., 1956.

[12] Naimark, M. A., About decomposition on factors-representations of unitary representation of locally compact group, Sibir. Mat. Jour. 2, 1(1961), 89-99.

[13] Naimark, M. A., About structure of factors-representations of locally compact group, DAN USSR 148(1963), 775-778.

[14] Rodriguez, A., Multiplicative characterization of Hilbert spaces and other interesting classes of Banach spaces, Rev. Mat. Univ. Complut. Madrid 9 (1996), 149-189.

[15] Naimark, M.A., On commuting unitary operators in spaces with indefinite metric, Acta. sci. szeged 24, 3-4(1963), 177-189.

[16] Dunford, N., Schwartz J. T., Linear operator, Part 1, General Theory, New York, London, 1958.

\section{Received: October 28, 2006}

